\title{
Unexpected hypersurfaces with multiple fat points
}

\author{
Justyna Szpond
}

October 1, 2019

\begin{abstract}
Starting with the ground-breaking work of Cook II, Harbourne, Migliore and Nagel, there has been a lot of interest in unexpected hypersurfaces. In the last couple of months a considerable number of new examples and new phenomena has been observed and reported on. All examples studied so far had just one fat point. In this note we introduce a new series of examples, which establishes for the first time the existence of unexpected hypersurfaces with multiple fat points. The key underlying idea is to study Fermat-type configurations of points in projective spaces.
\end{abstract}

\section{Introduction}

In the ground-breaking paper 4 the authors introduced the notion of unexpected curves.

Definition 1. We say that a reduced finite set of points $Z \subset \mathbb{P}^{2}$ admits an unexpected curve of degree $j+1$ if there is an integer $j>0$ such that, for a general point $P, j P$ fails to impose the expected number of conditions on the linear system of curves of degree $j+1$ containing $Z$. That is, $Z$ admits an unexpected curve of degree $j+1$ if

$$
h^{0}\left(\mathcal{J}_{Z+j P}(j+1)\right)>\max \left\{h^{0}\left(\mathcal{J}_{Z}(j+1)\right)-\left(\begin{array}{c}
j+1 \\
2
\end{array}\right), 0\right\} .
$$

This notion has been generalized to hypersurfaces in projective spaces of arbitrary dimension in the subsequent paper [7].

Definition 2. We say that a reduced set of points $Z \subset \mathbb{P}^{N}$ admits an unexpected hypersurface of degree $d$ if there exists a sequence of integers $m_{1}, \ldots, m_{s}$ such that for general points $P_{1}, \ldots, P_{s}$ the zero-dimensional subscheme $m_{1} P_{1}+\ldots+m_{s} P_{s}$ fails to impose independent conditions on forms of degree $d$ vanishing along $Z$.

A well known example of this kind is provided by 7 general double points in $\mathbb{P}^{4}$. There is a twisted quartic curve passing through these 7 points and its secant variety is a threefold of degree 3 vanishing to order 2 along the quartic curve, in particular in the 7 general points. This threefold is unexpected, see [1].

This example has two deficiencies. First, the singular points are not isolated. Second, the set $Z$ is empty.

The first example of an unexpected surface $Q_{R}$ in $\mathbb{P}^{3}$ admitted by a non-empty set $Z$ has been discovered and described in [2, Theorem 1]. In that example there is an isolated point $R$ of multiplicity 3 which is a general point. The surface $Q_{R}$ has 4 additional double points, whose coordinates depend on $R$, and no other singularities. In the present note we show the following result.

Keywords: fat points, linear systems, postulation problem, unexpected hypersurfaces, Fermat-type arrangements.

Mathematics Subject Classification (2010): MSC 14C20 MSC 14J26 MSC 14N20 MSC 13A15 MSC $13 \mathrm{~F} 20$. 
Main Theorem. There exists a non-empty set $Z$ in $P^{N}$ with $N=2 k+1$ for $k \geq 1$ which admits an unexpected hypersurface for multiplicities $3, \underbrace{2, \ldots, 2}_{k-1}$. This hypersurface has only isolated singularities.

For $N=3$ we have a computer-free proof. Our construction in higher dimensional projective spaces builds upon the existence of an unexpected surface of degree 4 in $\mathbb{P}^{3}$ with a general point of multiplicity 3 . Given a general point $R$ in $\mathbb{P}^{5}$ we project it to a coordinate $\mathbb{P}^{3}$ (i.e. codimension 2 flat $\Pi$ defined by the vanishing of two coordinates) obtaining the point $R_{\Pi}$. Then the $N=3$ case gives an unexpected surface in $\Pi$ which vanishes to order 3 at $R_{\Pi}$. Taking a cone in $\mathbb{P}^{5}$ with vertex in the coordinate line spanned by the coordinate points corresponding to the two coordinates which defined $\Pi$ we get a 4 -fold of degree 4 in $\mathbb{P}^{5}$ which in particular vanishes to order 3 at $R$. Of course this 4 -fold has non-isolated singularities. However, taking the linear system of quartics generated by all such cones in $\mathbb{P}^{5}$ we are able to identify its 6 generators. Given another general point $P$ in $\mathbb{P}^{5}$ we find an explicit formula for coefficients in front of the 6 generators (depending on $P$ ) so that the resulting 4 -fold has only isolated singularities and in particular has multiplicity 3 at $R$ and multiplicity 2 at $P$. Once the equation is in place, all our claims can be (in principle) checked by direct computation. We provide a Singular script [13] to ease their verification. The construction in higher dimensions is performed along the same lines. So far it justified only by computer computations with random points rather than arbitrary as in the case of Theorem 10.

The main idea here is to explore Fermat-type configurations of points in projective spaces, see [12] for a recent survey on Fermat-type hyperplane arrangements and their applications in algebra and geometry.

\section{Fermat-type point configurations}

By the way of warm-up we begin with points in $\mathbb{P}^{2}$. In Theorem 5 we show a peculiar family of unexpected curves, which degree grows while the multiplicity in the general point remains fixed, equal 4. Passing then to the general case of $\mathbb{P}^{N}$ we show identify generators of the ideal of Fermat-type configuration of points. This is crucial for the subsequent part.

\subsection{Fermat point configurations in $\mathbb{P}^{2}$}

Let $n \geq 1$ be a positive integer and let $I_{2, n}$ be the complete intersection ideal in $\mathbb{C}[x, y, z]$ viewed as the graded ring of $\mathbb{P}^{2}$ generated by

$$
x^{n}-y^{n}, y^{n}-z^{n} .
$$

Let $F_{2, n}$ be the ideal

$$
F_{2, n}=I_{2, n} \cap(x, y) \cap(x, z) \cap(y, z) .
$$

The support of $I_{2, n}$ is the set of $n^{2}$ points

$$
\left(1: \varepsilon^{\alpha}: \varepsilon^{\beta}\right)
$$

where $\varepsilon$ is a primitive root of unity or order $n$ and $1 \leq \alpha, \beta \leq n$. The support of $F_{2, n}$ is the union of the support of $I_{2, n}$ and the 3 coordinate points $(1: 0: 0),(0: 1: 0)$ and $(0: 0: 1)$.

The ideals $F_{2, n}$ have appeared recently in various guises, most notably in the context of the containment problem, see [11] for an introduction to this circle of ideas and [9], 8] for specific applications. Their algebraic properties have been studied in depth by Nagel and Seceleanu [10].

For $n=3$ the set of points defined by $F_{2,3}$ is the set of singular points (i.e. points where two or more arrangement lines meet) of the dual Hesse arrangement of 9 lines with 12 triple points at 


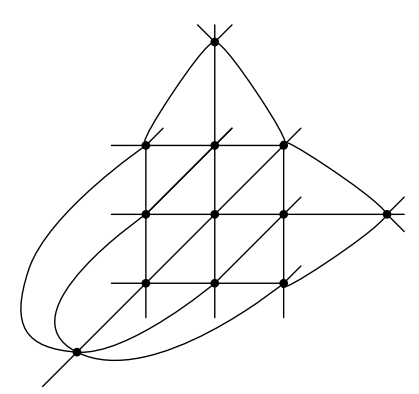

Figure 1: Dual Hesse arrangement

the zeroes of $F_{2,3}$. This arrangement is depicted in Figure 1, The lines are indicated by curved segments as it is not possible to embed this configuration in the real projective plane due to the celebrated Sylvester-Gallai Theorem, see [3]. It has been proved already in [6, Lemma 2.1] that the ideal $F_{2,3}$ is generated in degree 4 . More precisely we have

Lemma 3. The ideal $F_{2,3}$ is generated by the binomials

$$
x\left(y^{3}-z^{3}\right), y\left(z^{3}-x^{3}\right), z\left(x^{3}-y^{3}\right) .
$$

In fact exactly the same proof works for any $n \geq 3$, so that we have

Lemma 4. The ideal $F_{2, n}$ is generated by the binomials

$$
x\left(y^{n}-z^{n}\right), y\left(z^{n}-x^{n}\right), z\left(x^{n}-y^{n}\right) .
$$

This ideal is supported on $n^{2}$ points which form a complete intersection and the 3 coordinate points. It turns out that this set of points admits an unexpected curve (Definition 1) for any $n \geq 3$.

Theorem 5 (Unexpected curves with a point of multiplicity 4). Let $Z$ be the configuration of points in $\mathbb{P}^{2}$ defined by $F_{2, n}$ for $n \geq 3$. Let $R=(a: b: c)$ be a general point in $\mathbb{P}^{2}$. With $u=\left(\begin{array}{l}n \\ 2\end{array}\right)-1, \quad v=\left(\begin{array}{c}n-1 \\ 2\end{array}\right), \quad w=\left(\begin{array}{c}n+1 \\ 2\end{array}\right)$, the polynomial

$$
\begin{aligned}
Q_{P}(x: y: z)= & -c x y\left(\left(u b^{n}+v c^{n}\right)\left(z^{n}-x^{n}\right)+\left(u a^{n}+v c^{n}\right)\left(y^{n}-z^{n}\right)\right) \\
& -b x z\left(\left(u a^{n}+v b^{n}\right)\left(y^{n}-z^{n}\right)+\left(u c^{n}+v b^{n}\right)\left(x^{n}-y^{n}\right)\right) \\
& -a y z\left(\left(u b^{n}+v a^{n}\right)\left(z^{n}-x^{n}\right)+\left(u c^{n}+v a^{n}\right)\left(x^{n}-y^{n}\right)\right) \\
& +w a^{n-1} b c x^{2}\left(y^{n}-z^{n}\right)+w a b^{n-1} c y^{2}\left(z^{n}-x^{n}\right) \\
& +w a b c^{n-1} z^{2}\left(x^{n}-y^{n}\right)
\end{aligned}
$$

- vanishes at all points of $Z$,

- vanishes to order 4 at $R$,

- defines an unexpected curve of degree $n+2$ for $Z$ with respect to $R$.

Proof. This claim is easy verify by direct calculations, which we omit.

Theorem 5 is of interest as it exhibits a first family of unexpected curves, where the degree of curves grows but the multiplicity at the general point remains constant. 


\subsection{Fermat-type configurations of points in $\mathbb{P}^{N}$}

For a positive integer $n$ let $I_{N, n}$ be the complete intersection ideal defined by the binomials

$$
x_{0}^{n}-x_{1}^{n}, x_{1}^{n}-x_{2}^{n}, \ldots, x_{N-1}^{n}-x_{N}^{n} .
$$

The set $Z_{N, n}$ of zeroes of $I_{N, n}$ is the set of $N^{n}$ points of the form

$$
\left(1: \varepsilon^{\alpha_{1}}: \varepsilon^{\alpha_{2}}: \ldots: \varepsilon^{\alpha_{N}}\right)
$$

where $\varepsilon$ is a primitive root of unity of order $n$ and

$$
1 \leq \alpha_{1}, \alpha_{2}, \ldots, \alpha_{N} \leq n
$$

Adding to this set of points the coordinate points of $\mathbb{P}^{N}$, we obtain the set $W_{N, n}$ defined by the ideal $F_{N, n}$.

In the sequel we will deal with the index $n=3$, so we drop the degree from the notation. We also use the convention that the indices are understood modulo $N+1$. For example $x_{N+2}=x_{1}$. Moreover it is convenient to introduce the following notation

$$
[a, b]=a^{3}-b^{3},
$$

which we use for numbers and for monomials. Note that the symbol $[a, b]$ is anti-symmetric and satisfies a Jacobi-type identity

$$
a^{3}[b, c]+b^{3}[c, a]+c^{3}[a, b]=0 \text { for all } a, b, c .
$$

Lemma 3 generalizes in the following way.

Lemma 6. The ideal $F_{N}$ is generated by the binomials of the form

$$
x_{i}\left[x_{i+1}, x_{j}\right],
$$

where $i \in\{0,1, \ldots, N\}$ and $j \in\{0,1, \ldots, N\} \backslash\{i, i+1\}$.

Proof. Let $J$ be the ideal generated by binomials in (4). It is clear that elements in $J$ vanish in all points of $W_{N}$, so there is $J \subset F_{N}$.

It is also easy to see that $J$ contains all binomials of the form $x_{i}\left[x_{j}, x_{k}\right]$, where the indices are mutually distinct. Indeed, we have

$$
x_{i}\left[x_{j}, x_{k}\right]=x_{i}\left[x_{i+1}, x_{k}\right]-x_{i}\left[x_{i+1}, x_{j}\right] .
$$

It is also clear that the ideal $I_{N}$ defined in (2) is generated also by binomials

$$
\left[x_{0}, x_{1}\right],\left[x_{0}, x_{2}\right], \ldots,\left[x_{0}, x_{N}\right],
$$

which are slightly more convenient to work with in this proof.

Let $f \in F_{N}$ be an arbitrary polynomial. We want to show the containment $f \in J$ or equivalently $f=0 \bmod J$. Since $f$ vanishes in particular in all points of $Z_{N}$, there are homogeneous polynomials $g_{1}, \ldots, g_{N}$ such that

$$
f=\sum_{i=1}^{N} g_{i}\left[x_{0}, x_{i}\right] .
$$

from now on, we work $\bmod J$. Since for $j \neq 0, i$ the binomials $x_{j}\left[x_{0}, x_{i}\right]$ are in $J$, we may assume that each polynomial $g_{i}$ depends only on $x_{0}$ and $x_{i}$. Moreover, for a fixed $j \neq 0, i$ we have

$$
x_{0} x_{i}\left[x_{0}, x_{i}\right]=x_{i} \cdot x_{0}\left[x_{j}, x_{i}\right]-x_{0} \cdot x_{i}\left[x_{j}, x_{0}\right] \in J .
$$


Thus it must be

$$
g_{i}=a_{i} x_{0}^{d}+b_{i} x_{i}^{d} \bmod J
$$

for some $d \geq 0$ and some scalars $a_{1}, \ldots, a_{N}, b_{1}, \ldots, b_{N} \in \mathbb{C}$. Evaluating (5) in the coordinate points we obtain

$$
a_{1}+a_{2}+\ldots+a_{N}=0 \text { and } b_{1}=b_{2}=\ldots=b_{N}=0 .
$$

Hence (5) reduces to

$$
f=a_{1} x_{0}^{d}\left[x_{0}, x_{1}\right]+a_{2} x_{0}^{d}\left[x_{0}, x_{2}\right]+\ldots+a_{N} x_{0}^{d}\left[x_{0}, x_{N}\right] \bmod J .
$$

If $d=0$, then evaluating (7) in the coordinate points, we obtain

$$
a_{1}=a_{2}=\ldots=a_{N}=0
$$

and we are done.

If $d>0$, then, using first part of (6), we can rewrite (17) as

$$
f=a_{1} x_{0}^{d}\left[x_{N}, x_{1}\right]+a_{2} x_{0}^{d}\left[x_{N}, x_{2}\right]+\ldots+a_{N-1} x_{0}^{d}\left[x_{N}, x_{N-1}\right],
$$

which is clearly an element of $J$ and we are done again.

\section{Unexpected quartic surfaces in $\mathbb{P}^{3}$ with a triple point}

The story of unexpected hypersurfaces begins with unexpected curves discovered by Di Gennnro, Ilardi and Vallès in [5] and studied systematically by Cook II, Harbourne, Migliore and Nagel in [4]. In 2] the first example of a higher dimensional unexpected hypersurface (a surface in $\mathbb{P}^{3}$ ) has been described by Bauer, Malara, Szemberg and the present author. Shortly after that Harbourne, Migliore, Nagel and Teitler in [7] constructed examples of unexpected hypersurfaces in projective spaces of arbitrary dimension. All these examples have just one singular general point.

Here we recall the construction of the unexpected quartic in $\mathbb{P}^{3}$. This example has not only motivated our construction of unexpected hypersurfaces with multiple fat points but it serves as a building fundament for this construction.

According to Lemma 6 , in $\mathbb{P}^{3}$ the ideal $F_{3,3}$ has 8 generators:

$$
\begin{aligned}
& g_{0,2}=x_{0}\left[x_{1}, x_{2}\right], g_{0,3}=x_{0}\left[x_{1}, x_{3}\right], g_{1,3}=x_{1}\left[x_{2}, x_{3}\right], g_{1,0}=x_{1}\left[x_{2}, x_{0}\right], \\
& g_{2,0}=x_{2}\left[x_{3}, x_{0}\right], g_{2,1}=x_{2}\left[x_{3}, x_{1}\right], g_{3,1}=x_{3}\left[x_{0}, x_{1}\right], g_{3,2}=x_{3}\left[x_{0}, x_{2}\right] .
\end{aligned}
$$

It is convenient to introduce the following notation. Given mutually distinct numbers $i, j \in$ $\{0,1,2,3\}$, we denote by $k$ the index $\{0,1,2,3\} \backslash\{i, i+1, j\}$. As usually, the indices are considered modulo 4. With this notation we have (compare [2, Theorem 6]).

Theorem 7. For a general point $R=\left(a_{0}: a_{1}: a_{2}: a_{3}\right)$, the quartic

$$
Q_{R}\left(x_{0}: x_{1}: x_{2}: x_{3}\right)=\sum_{i=0}^{3} \sum_{j=i+2}^{i+3}(-1)^{k} a_{i}^{2}\left[a_{i+1}, a_{k}\right] \cdot g_{i, j}
$$

has a triple point at $R$ and satisfies $Q_{R} \in F_{3,3}$.

Proof. The second property is obvious. Checking that $Q_{R}$ vanishes to order 3 at $R$ is a straightforward computation. However for further reference we will present some computations which make heavy use of available symmetries. 


$$
\left(\begin{array}{cccccccc}
0 & 0 & -a_{1} & 0 & -a_{2} & 0 & a_{3} & a_{3} \\
a_{1}^{2} & a_{1}^{2} & -a_{0}^{2} & 0 & 0 & 0 & 0 & 0 \\
a_{2}^{2} & 0 & 0 & 0 & a_{0}^{2} & 0 & 0 & 0 \\
0 & -a_{3}^{2} & 0 & 0 & 0 & 0 & a_{0}^{2} & a_{0}^{2} \\
a_{0} & a_{0} & 0 & 0 & 0 & -a_{2} & -a_{3} & 0 \\
0 & 0 & a_{2}^{2} & a_{2}^{2} & 0 & -a_{1}^{2} & 0 & 0 \\
0 & 0 & 0 & a_{3}^{2} & 0 & 0 & a_{1}^{2} & 0 \\
-a_{0} & 0 & a_{1} & a_{1} & 0 & 0 & 0 & -a_{3} \\
0 & 0 & 0 & 0 & a_{3}^{2} & a_{3}^{2} & 0 & -a_{2}^{2} \\
0 & -a_{0} & 0 & -a_{1} & a_{2} & a_{2} & 0 & 0
\end{array}\right)
$$

Table 1: Interpolation matrix for a point of multiplicity 3 in $\mathbb{P}^{3}$.

To begin with, we check that the first order derivatives of $Q_{R}$ vanish at $R$. By symmetry it is enough to check just one derivative. We compute the one with respect to $x_{0}$ and obtain

$$
\frac{\partial}{\partial x_{0}} Q_{R}=\left(\left[a_{1} a_{2}\right]\left[x_{1} x_{3}\right]-\left[a_{1} a_{3}\right]\left[x_{1} x_{2}\right]\right) a_{0}^{2}+3 x_{0}^{2}\left(a_{1}^{2} x_{1}\left[a_{2} a_{3}\right]+a_{2}^{2} x_{2}\left[a_{3} a_{1}\right]+a_{3}^{2} x_{3}\left[a_{1} a_{2}\right]\right) .
$$

Vanishing of the first bracket when evaluating at $R$ is straightforward. The vanishing of the second bracket follows from the Jacobi-type identity (3).

For the second order derivative with respect to $x_{0}$ we have

$$
\frac{\partial^{2}}{\partial x_{0}^{2}} Q_{R}=-6 x_{0} \cdot \sum_{k=1}^{3}(-1)^{k} a_{j}^{2} a_{l}^{2}\left(a_{l} x_{j}-a_{j} x_{l}\right),
$$

where the indices $j, l$ are chosen in dependence on $k$ so that $j<l$ and $\{j, k, l\}=\{1,2,3\}$.

For the mixed derivative of order 2 we may again use the symmetry and look at just one other variable, here $x_{1}$. We have

$$
\frac{\partial^{2}}{\partial x_{1} \partial x_{0}} Q_{R}=3\left[a_{2} a_{3}\right]\left(a_{1}^{2} x_{0}^{2}-a_{0}^{2} x_{1}^{2}\right) .
$$

Clearly, in both cases we obtain zero, when evaluating at $R$.

Instead of computing derivatives one by one, we can also use the whole interpolation matrix at once. We present this matrix in Table 1, since this is a baby case for our argument in the proof of Theorem 10, see Table 2, The columns of the table correspond to the generators of $F_{3,3}$, whereas the rows stand for order 2 partial derivatives. Taking into account that the point $R$ is general, we have divided out common factors appearing in some rows. This is the reason why not all rows have entries of the same degree. Nevertheless, the matrix as entire, remains of course homogeneous.

\section{Unexpected quartic 4 -folds in $\mathbb{P}^{5}$ with a triple and double point}

In this section we present in detail unexpected hypersurfaces in $\mathbb{P}^{5}$, which have 2 general fat points, one of multiplicity 3 and the other one of multiplicity 2 .

According to Lemma 6 the ideal $F_{5,3}$ has 24 generators and its zero locus consists of $3^{5}$ Fermat points and 6 coordinate points, so that there are altogether 249 points in $W_{5,3}$. The linear system of quartics in $\mathbb{P}^{5}$ has (affine) dimension 126 , so the points in $W_{5,3}$ do not impose independent conditions on quartics. Of course there exists a subset of 102 points in $W_{5,3}$ which impose independent conditions on quartics in $\mathbb{P}^{5}$ but to determine such a set is not relevant for our considerations here. 
Since vanishing to order 3 imposes 21 conditions, it is expected that the system of quartics in $\mathbb{P}^{5}$ vanishing along $W_{5,3}$ and having a triple point in a general point $R=\left(a_{0}: a_{1}: \ldots: a_{5}\right)$ has dimension 3. It turns out however that this system has in fact higher dimension.

Proposition 8. Let $I(R)$ denote the ideal of the point $R$. For

$$
V_{5 ; 3}=H^{0}\left(\mathbb{P}^{5} ; \mathcal{O}_{\mathbb{P}^{5}}(4) \otimes F_{5,3} \otimes I(R)^{3}\right)
$$

we have $\operatorname{dim} V_{5 ; 3}=6$.

Proof. We verify our claim computing the interpolation matrix at $R$. More precisely, we compute all partial derivatives of order 2 (there are 21 of them) of all 24 generators of $F_{5,3}$. The matrix we get has a lot of zeros and thus its rank is easy to determine. The rank is the number of independent conditions imposed on quartics in $F_{5,3}$ by vanishing at $R$ to order 3 . 


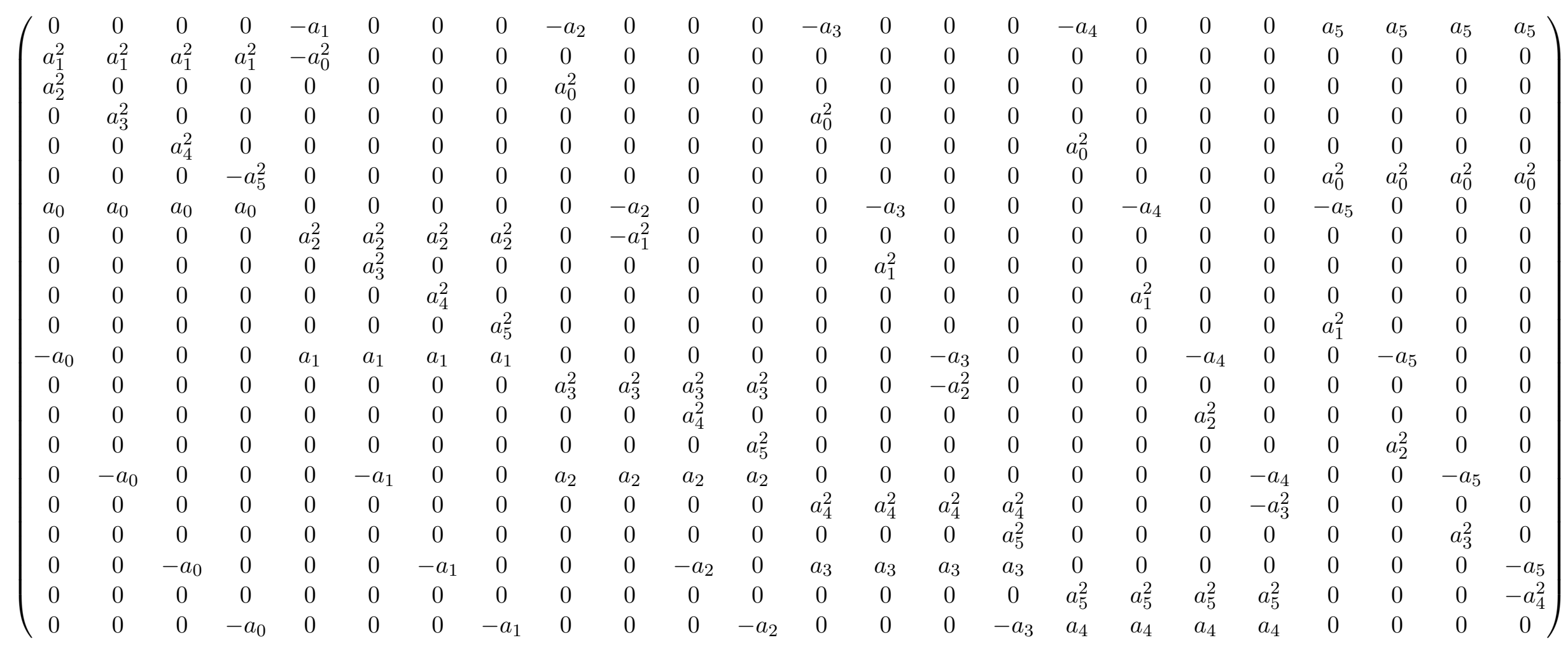

Table 2: Interpolation matrix for a point of multiplicity 3 in $\mathbb{P}^{5}$. 
Since a double point in $\mathbb{P}^{5}$ imposes 6 conditions on forms of arbitrary degree, we do not expect that there is a non-zero section in $V_{5 ; 3}$ vanishing to order 2 at an additional general point. However such unexpected hypersurface does exist!

We define first the following 6 cones in $\mathbb{P}^{5}$. For indices $i, j \in\{0, \ldots, 5\}$, with $i<j$, we denote by $s, t, u, v$ the remaining 4 indices in the growing order. Then, for $i, j \in\{0,1,2,3\}$ with $i<j$ we set

$$
R_{i, j}=\left(a_{s}: a_{t}: a_{u}: a_{v}\right)
$$

and

$$
J_{i, j}=Q_{R_{i, j}}\left(x_{s}: x_{t}: x_{u}: x_{v}\right),
$$

where $Q_{R}$ is taken from Theorem 7

Remark 9. It can be shown that $J_{0,1}, \ldots, J_{2,3}$ generate $V_{5,3}$ but this is irrelevant for the further assertions and we omit the verification of this claim.

Theorem 10. Let $P=\left(b_{0}: b_{1}: \ldots: b_{5}\right)$ be a general point in $\mathbb{P}^{5}$. Then there exists a unique quartic $Q_{R, P} \in V_{5 ; 3}$ vanishing at

- all points of the Fermat-type configuration $W_{5}$,

- the point $R=\left(a_{0}: a_{1}: \ldots: a_{5}\right)$ to order 3 ,

- the point $P$ to order 2.

Proof. We are able to write down explicit equation of the quartic

$$
\begin{aligned}
Q_{R, P}\left(x_{0}: \ldots: x_{5}\right) & =J_{2,3}(P) \cdot J_{0,1}\left(x_{0}: \ldots: x_{5}\right)-J_{1,3}(P) \cdot J_{0,2}\left(x_{0}: \ldots: x_{5}\right) \\
& +J_{0,3}(P) \cdot J_{1,2}\left(x_{0}: \ldots: x_{5}\right)+J_{1,2}(P) \cdot J_{0,3}\left(x_{0}: \ldots: x_{5}\right) \\
& -J_{0,2}(P) \cdot J_{1,3}\left(x_{0}: \ldots: x_{5}\right)+J_{0,1}(P) \cdot J_{2,3}\left(x_{0}: \ldots: x_{5}\right) .
\end{aligned}
$$

The sign in the front of a summand of $Q_{R, P}$ depends on whether there is a pair of consecutive numbers in the indices of involved $J$ 's or not. If there isn't, i.e., for pairs $(0,2)$ and $(1,3)$ we get a minus.

Note that the first two assertions of the Theorem follow straightforward from the way we defined the cones $J_{0,1}, \ldots, J_{2,3}$. The last assertion can be checked by direct computations as in the proof of Theorem 7 .

The most tricky part of the proof was to find the coefficients in front of the cones.

\section{Unexpected quartic $2 k$-folds in $\mathbb{P}^{2 k+1}$ with a triple point and $k-1$ double points}

Before we conclude we collect a couple of facts towards the proof of our Main Theorem. We begin with an observation which parallels Proposition 8

Proposition 11. Let $N=2 k+1$ for some $k \geq 1$. Let $R=\left(a_{0}: \ldots: a_{N}\right)$ be a general point in $\mathbb{P}^{N}$ and let $I(R)$ be its saturated ideal. Let

$$
V_{N, 3}=H^{0}\left(\mathbb{P}^{N} ; \mathcal{O}_{\mathbb{P}^{N}}(4) \otimes F_{N, 3} \otimes I(R)^{3}\right) .
$$

Then $\operatorname{dim} V_{N, 3}=\left(\begin{array}{c}N-1 \\ 2\end{array}\right)$.

Remark 12. The statement of Proposition 11 means that a general triple point always imposes exactly 3 conditions less on generators of $F_{N, 3}$ than expected. We don't know how to verify this theoretically.

The final step in proving Main Theorem is the next statement. 
Proposition 13. Let $P_{1}, \ldots, P_{k-1}$ be general points in $\mathbb{P}^{N}$ with $N=2 k+1$. Vanishing to order 2 in these points imposes $2 k^{2}-k-1$ conditions on elements in $V_{N, 3}$.

Remark 14. Note that vanishing to order 2 at a general point is expected to impose $N+1=$ $2 k+2$ conditions. Thus $(k-1)$ points are expected to impose $2 k^{2}-2$ conditions. Interestingly, computer experiments show that the first $(k-2)$ points do impose on $V_{N, 3}$ the expected number of conditions, namely $2 k^{2}-2 k-4$. It is only the last point which imposes $k+3$ instead of $2 k+2$ conditions on the system

$$
H^{0}\left(\mathbb{P}^{N} ; \mathcal{O}_{\mathbb{P}^{N}}(4) \otimes F_{N, 3} \otimes I(R)^{3} \otimes I\left(P_{1}\right)^{2} \otimes \ldots \otimes I\left(P_{k-2}\right)^{2}\right) .
$$

We do not have a theoretical explanation of this phenomena.

\section{References}

[1] J. Alexander and A. Hirschowitz. Polynomial interpolation in several variables. J. Alg. Geom., 4(2):201-222, 1995.

[2] T. Bauer, G. Malara, T. Szemberg, and J. Szpond. Quartic unexpected curves and surfaces. Manuscripta Math., to appear, doi.org/10.1007/s00229-018-1091-3.

[3] P. Borwein and W. O. J. Moser. A survey of Sylvester's problem and its generalizations. Aequationes Math., 40(2-3):111-135, 1990.

[4] D. Cook II, B. Harbourne, J. Migliore, and U. Nagel. Line arrangements and configurations of points with an unexpected geometric property. Compos. Math., 154(10):2150-2194, 2018.

[5] R. Di Gennaro, G. Ilardi, and J. Vallès. Singular hypersurfaces characterizing the Lefschetz properties. J. Lond. Math. Soc. (2), 89(1):194-212, 2014.

[6] M. Dumnicki, T. Szemberg, and H. Tutaj-Gasińska. Counterexamples to the $I^{(3)} \subset I^{2}$ containment. J. Algebra, 393:24-29, 2013.

[7] B. Harbourne, J. Migliore, U. Nagel, and Z. Teitler. Unexpected hypersurfaces and where to find them, arXiv:1805.10626, accepted for publication in Michigan Math. J.

[8] G. Malara and J. Szpond. Fermat-type configurations of lines in $\mathbb{P}^{3}$ and the containment problem. J. Pure Appl. Algebra, 222(8):2323-2329, 2018.

[9] G. Malara and J. Szpond. On codimension two flats in Fermat-type arrangements. In Multigraded Algebra and Applications, Springer Proceedings in Mathematics \& Statistics, pages 95-109. Springer, 2018.

[10] U. Nagel and A. Seceleanu. Ordinary and symbolic Rees algebras for ideals of Fermat point configurations. J. Algebra, 468:80-102, 2016.

[11] T. Szemberg and J. Szpond. On the containment problem. Rend. Circ. Mat. Palermo (2), 66(2):233-245, 2017.

[12] J. Szpond. Fermat-type arrangements, arXiv:1909.04089.

[13] J. Szpond. Singular file, http://szpond.up.krakow.pl/jusF.

Justyna Szpond, Department of Mathematics, Pedagogical University of Cracow, Podchorążych 2, PL-30-084 Kraków, Poland.

E-mail address: szpond@gmail.com 